\title{
Succulent Incubator Design with Automatic Adjustment Function
}

\author{
Gao Lixia, Shen Hanlin \\ Civil Aviation Flight University of China, China \\ *gaolx0129@126.com
}

Keywords: Arduino; humidity monitoring; illumination adjusting; DHT11

\begin{abstract}
More and more people like succulents because of its unique ornamental.Their growth state and shape depend on the growth environment. The specific requirement of the environment includes the temperature, light and humidity. Suitable environmental conditions can develop highly ornamental value products. Here the incubator design accords to the three aspects of the environment needs, using the intelligent and manual control, to optimize the culture environment of the succulents, to meet the individual needs of the culture environment enthusiasts at a certain extent. The incubator has an alarm function, if the temperature and humidity exceeds or falls below the set value, the alarm is triggered. The heater will automatically heat. Meanwhile, the light intensity is also be monitored. When the value is below the set value, it can automatically be adjusted to keep it near the set value. The incubatorcan satisfy the necessary growth conditions of succulents, to achieve the purpose of the design.
\end{abstract}

\section{Demand situation of incubator}

Nowadays,personalized and fresh succulents become home and office consumers'darling. Succulents have many different varieties and different colors. It is very convenient to place them in the room or on the balcony. And it also has the function to purify the air, so more and more people fall in love with them.

In the plant growth environment, temperature, moisture, light, soil, air and other factors are important to them. The plant growth and development are influenced by the factors above. That means that they will have an important ecological role. Cultivate succulents soil has its unique material ratio, in accordance with the ratio requirements it is very easy to implementwith the soil. But the temperature, light, moisture and other factors are limited by the natural growth environment.Thus, the succulent bought from the gardening seller is easy to be broken or longer ugly during the growing period, which make the interest growers to lose their confidence and enthusiasm.

Thus, to design a small incubator which can provide an ideal culture environment for thesucculent is very useful. This small incubator can meet the basic needs of the culture enthusiasts.

\section{Function of the incubator and main device selection}

Succulents like hot environment, not like the cold environment, the growth temperature of them is not less than 5 degrees Celsius. The optimum growth temperature is between 25-30 degrees Celsius. They do not like much water. The optimum growthhumidity is at about $40 \%$. It does not require frequent watering. They like sunshine and need grow in sunny zone.The light intensity maintained at about 300lx is good. According to the needs analysis, the function of the 
incubatorincludes temperature control, humidity detection and alarm, lighting control, parameter displays and adjustment.

In this system, DHT11 temperature and humidity sensor is selected for detecting the temperature and humidity in the incubator. BH1750FVI is used as light sensors, to finish acquisition of the illumination data. A $320 * 480$ serial port touch color display module is used to display real-time temperature and lighting conditions. Arduino is selected as the processingplatform, which can meet the data acquisition speed. The basic structureblock diagram of the system is shown in figure 1 .

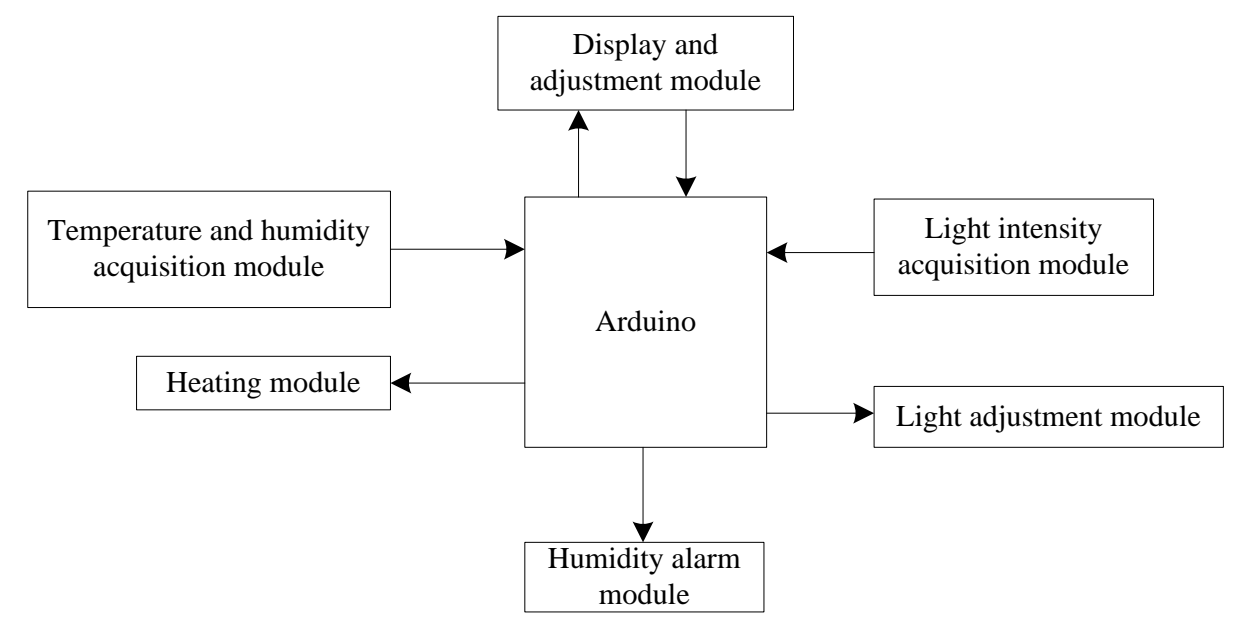

Figure 1. Overall block diagram of the system hardware circuit

\section{Incubator hardware circuit design}

\subsection{Working platform}

Arduino is an open-source electronics prototyping platform, which is convenient, flexible and easy-to-use. It contains the hardware (various models of the Arduino board) and software (Arduino IDE).

Arduino IDE can run on three major operating systems: Windows, Macintosh OS X and Linux. It development is based on processing IDE, which is easy to grasp, and has enough flexibility. Arduino hardware schematic diagrams, schematics, IDE software and core libraries are open source. Within the scope of the agreement we can modify the original design and the corresponding codearbitrarily.

\subsection{Light sensor and work circuit}

Light sensor is divided into two categories: type photosensitive resistance and highly integrated digital light sensors. Integrated light sensor can be adjusted according to the brightness of the light intensity, and it has high resolution, relative large measuring range. This type sensor does not need complex external circuit in specific design, so it is selected in this design, model BH1750FVI.This sensor has an internal 16-bit ADC and the output is a digital signal directly. So it does not need some complex calculations here. It has five pins: VCC, SCL, SDA, ADDR and GND.Wherein, VCC connected to the power supply voltage of $5 \mathrm{~V}$, SCL is the clock bus pinof IIC, SDA is the data bus pinof IIC, ADDR is the address pinIIC device. Specific operation circuit is shown in figure 2. 


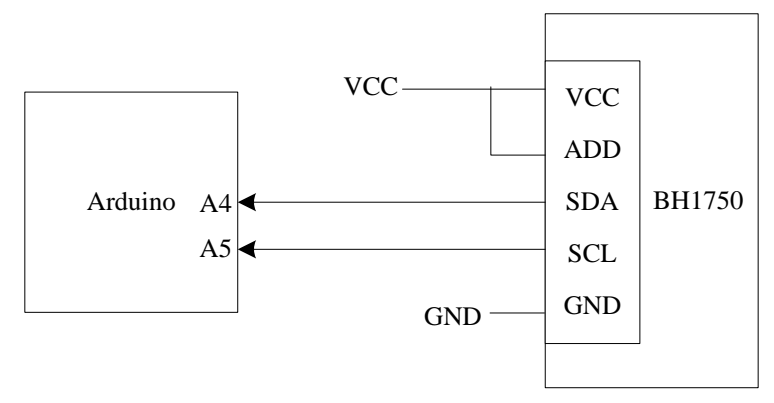

Figure 2. Connect between arduino and BH1750

SDA and SCL ports of BH1750 are connected toanalog A4and A5 ports of arduino. The value got by Arduino is the last result of the measurement on the sensor. BH1750 has very high accuracy andvery strong sensitivity, we can take the measurement value as the true value.

\subsection{Light adjustment circuit}

LED has many characteristics, such as low power, strong power units and so on.The light emitting from it includes the red, blue band optical fiber, these fibers is helpful to the growth of the succulent, including plant growth red, light blue band, as long as the power is enough to ensure plant growth. The Lights are assembled on the band-like FPC (flexible printed circuit board) or PCB hard board to constitute lampstrip. This lamp strip has good flexibility, is free to bend and has waterproof performance characteristics, so it is selected to be used in this design to generating light. Its rated voltage is $12 \mathrm{~V}$, rated power is $18 \mathrm{~W}$, LED lights single lumen is about 40LM $\sim 45 \mathrm{LM}$, identified life is 50,000 hours. Light adjustment working principle circuit is shown in figure 3.

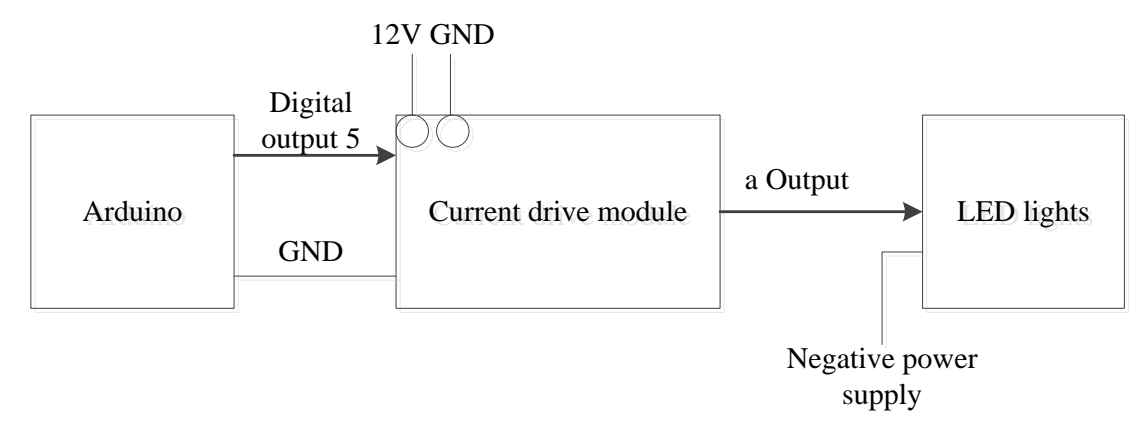

Figure 3. Light adjusting working principle circuit

PWM module port 5 of arduino is connected to the drive input module. When the light above a given value, the analog voltage from the PWM output port is reduced, through the driver module, the voltage obtained by LED lights is reduced accordingly. LED lights are dimmed, light intensity decreases. When the light is below a given value, the analog voltage from the PWM output port isincreased, through the driver module.The voltage obtained by LED lights is increased accordingly.LED lights are brighten, light intensity increases. Closed-loop control is used in this system to maintain the illumination substantially equals the given value.

\subsection{Temperature and humidity sensors operating circuit}

Temperature and humidity sensor is a type of sensitive element that can sense temperature and humidity of the measurement environment. This kind of sensor includes analog output temperature and humidity sensors, logic-output temperature and humidity sensors, digital-output temperature and humidity sensors. Digital-output temperature and humidity sensor's output signal is a digital signal directly, therefore do not need A/D conversion, and has strong anti-interference ability, without an 
external amplifier, the external circuit is very simple. So according to the specific requirements of the system, DHT11 temperature and humidity sensor is selected in this system. DHT11 has 4pins: VCC, DATA, N/A, and GND.Wherein, VCC voltage is 3.5-5V. It needs to notice that when using $3.5 \mathrm{~V}$ voltage supply, cable length should not be greater than $20 \mathrm{~cm}$. Because the line drop can lead to insufficient power the sensor, resulting in measurement error.

DATA is a bi-directional interface for serial communication and synchronization between the microprocessor and DHT11, single bus data format. In each communication,40-bit data are transferred in accordance with the high first-out order, the time be used about 4ms. Data format is 8-bit humidity integer data,8-bit humidity decimal data,8-bit temperature integer data,8-bit temperature decimal data. The useful data is integer data, and the decimal data is all zero. If the data transfer is correct, the checksum data is equal to the last eight bits of the sum of "8-bit humidity integer data +8 -bit humidity data decimal +8 -bit temperaturedecimal data +8 -bit temperature decimal data".

\subsection{Heater work circuit}

In this system, PTC heating film is used for heating. It consists of several chips and corrugated aluminum cooling. The high temperature adhesive is used to put them together.This heater has advantages of small thermal resistance and high heat transfer efficiency. Its biggest feature is a high security, because the surface temperature of the heater will maintain limited around Curie temperature (usually $220{ }^{\circ} \mathrm{C}$ ). At the same time, in order to spread the heat throughout the incubator, a cooling fan is used in the heating system to help dissipate heat. Temperature monitoring principle circuit is shown in figure 4.

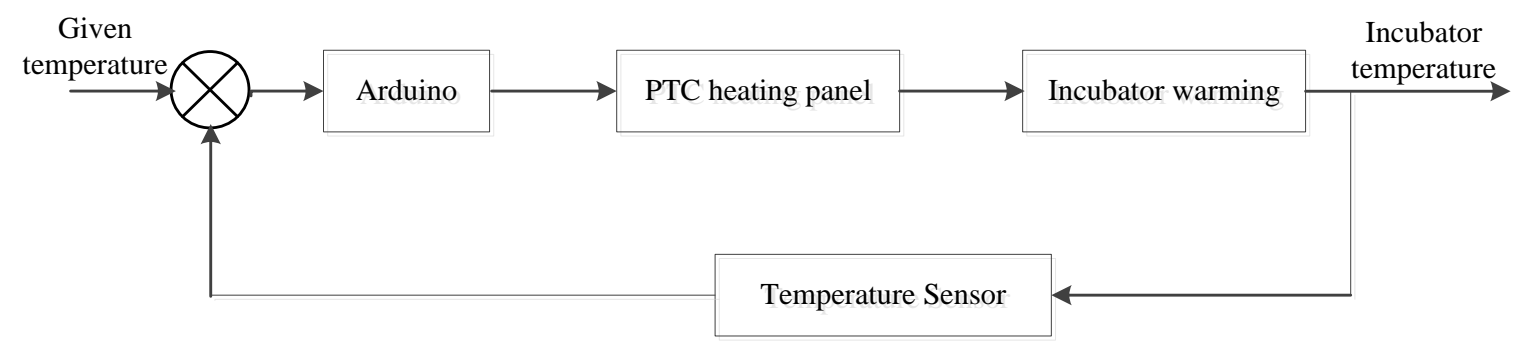

Figure 4. Temperature monitoring works circuit

\subsection{Serial port touch color screen}

The serial port touch color screen is used in this design to display the temperature and humidity or adjust the thresholdof the temperature and humidity. This deviceintegrates the regulation and display functions, so it can simplify the system and is convenient to program. The module has four pins, + $5 \mathrm{~V}$ and GND are power supply pins. RX is thedata receive pin of the serial port screen, which is connected to the RX port of arduino. TX screen is serial data transmission pin of theserial port touch color screen, which isconnected to the TX port of arduino.

\subsection{Incubator cabinet design}

Acrylic is also called special treatment organic glass, which is organic glassreplacement. Theincubator is made with acrylic has many advantages, such as good light transmission, pure color, rich colors and beautiful formation, long life, and so on. In addition, the acrylic sheet can perfect combinedwith plate aluminum profiles, advanced screen printing, etc. So, this material is used to make the box. 


\section{System function program}

Hardware circuit works under the driver of the software program. The software programs is used to collecting data from the various sensors and make the judgmentaccording to the set parameter value to determine the adjustment of the temperature, light and humidity in the incubator. So, in addition to the main program, there are a number of functional subroutines, including monitoring data reading module program, the displays module program, execution module program,etc.

\section{Actual functional testing and implementation}

During the process of the system design, the typical device functional test and the various typical circuits test are finished. After this, the overall system function is tested. The functions of humidity monitoring display, temperature monitoring display and adjustment, lighting monitoring display and adjustmentare well realized, to meet the initial target of the design.

\section{Reference}

[1] DanaoHan, Fei Wang 2013 Applied research of DHT11 digital temperature and humidity sensors Electronic Design Engineering

[2] Changsheng Liu 2012Sensor cookbook and application circuit-temperature sensor volume( Xi'an: Xi'an University Press)

[3] Yue Zhang, Jing Pan, Weidong Sun, Huiqiong Zheng 2015 Influence of LED spectrum on growth and development of plants in the simulation space incubatorSpace Science News

[4] Lvzhou Chen 2015 Arduino programming foundation (Beijing: Beijing University of Aeronautics and Astronautics Press)

[5] Michael Margolis 2015 Arduino Definitive Guide (2nd Edition) Yang Kunyun (Beijing: People's Posts and Telecommunications Press)

[6] Danfeng Zhao, Xin Liu 2013 translated integrated temperature sensor multi-point temperature measurement should sensors technology (Beijing: Science Press) 